\title{
Perioperative management of a patient with Automated Implantable Cardioverter Defibrillator (AICD)
}

\author{
Sukanya Mitra, Vanita Ahuja, Anudeep Jafra, Suman Shekhawat \\ Government Medical College and Hospital \\ Sector 32, Chandigarh, India.
}

*Corresponding author: drsmitra12@yahoo.com

Key words: AICD; anaesthesia; perioperative; management

\begin{abstract}
Perioperative electromagnetic interference and their potential effects on automated implantable cardioverter defibrillator (AICD) pose a considerable challenge to anaesthesiologists. A 73year-old male weighing $30 \mathrm{~kg}$ with fracture neck of femur and with a background history of hypertension, severe left ventricular dysfunction and coronary artery disease with an implanted AICD in situ was scheduled for left sided hemiarthroplasty. The AICD was disabled during surgery. He received graded epidural with bupivacaine and fentanyl. He also received continuous epidural anaesthesia for postoperative pain relief. He had an uneventful perioperative period. We review this area and discuss the perioperative anaesthetic challenges in such patients.
\end{abstract}

The number of geriatric patients with cardiac electrophysiological disorders for non cardiac surgery is globally on a rise due to increasing availability of better medical facilities and diagnostic methods. ${ }^{1}$ Those with low ejection fraction do not respond to medical therapy hence receive automated implantable cardioverter defibrillator $\quad$ (AICD) ${ }^{1} \quad$ Perioperative electromagnetic interference and their potential effects on AICD pose a considerable challenge to the anaesthesiologists. ${ }^{2} \mathrm{We}$ present a case of a patient with severe left ventricular dysfunction with implanted pacemaker with AICD posted for hemiarthroplasty and discuss the anaesthetic challenges in such patients.

\section{Case report}

A 73-year-old male weighing $30 \mathrm{~kg}$ with fracture neck of femur and with a background history of hypertension and coronary artery disease with an implanted AICD (Epic ${ }^{\mathrm{TM}}$ Model V-338, St Jude Medical, Sylmar, CA, USA) in situ was scheduled for left sided hemiarthroplasty. The AICD had right ventricle paced, sensed and inhibited (VVI) as permanent modes with anti-tachycardia with bradycardia pacing. He was a known hypertensive for last ten years and had an inferior wall myocardial infarction (MI) seven years back. He also had an anterior wall MI two years back following which an AICD was implanted. Since then he had no episodes of angina, dyspnoea or AICD malfunction. The patient received daily oral preparations of ramipril $2.5 \mathrm{mg}$, amiodarone 200 $\mathrm{mg}$ and aspirin $150 \mathrm{mg}$ along with low molecular weight heparin (LMWH) $0.4 \mathrm{ml}$ subcutaneously once a day. He also received oral isosorbide mononitrate $10 \mathrm{mg}$ and metoprolol $25 \mathrm{mg}$ twice a day.

On examination, the patient had a regular pulse rate of 50 per minute with blood pressure of $130 / 80 \mathrm{mmHg}$. His room air saturation was $95 \%$. Other than the preoperative haemoglobin of 10 $\mathrm{g} / \mathrm{dl}$ his other haematological and biochemical investigations were within normal limits. The electrocardiogram showed left bundle branch block with ST segment elevation in V1-V6. Preoperative echocardiography showed severe left ventricle systolic dysfunction, with ejection fraction of $20 \%$ with mild mitral and aortic regurgitation. AICD was reviewed by the company designated programmer for evaluating battery status and adequacy of performance under current settings. Per his advice it was planned to deactivate AICD during the intraoperative period using a magnet. The device was set to an asynchronous mode and heart rate was set at 72 beats /minute. 
All medications were continued till the day of surgery except aspirin, which was stopped one week prior to surgery. LMWH was also stopped 24 hours prior to surgery. The patient received premedication of oral ranitidine $150 \mathrm{mg}$ and alprazolam $0.25 \mathrm{mg}$ on the night before surgery and two hours prior to surgery. In the operating room, 20G intravenous line was secured in left hand and he was monitored for non invasive blood pressure, heart rate, pulse oximetry, ECG and urine output. With all aseptic precautions invasive arterial line was secured in right radial artery under local anaesthesia. AICD was deactivated; sterile external pacing pads were attached and defibrillator was kept ready in the operating room. An 18 G epidural catheter was inserted in L3- L4 interspace with the patient in the sitting position. After a $3 \mathrm{ml}$ test dose of $2 \%$ lignocaine hydrochloride, graded epidural with aliquots of 5 $\mathrm{ml}$ of $0.5 \%$ bupivacaine with fentanyl was injected to achieve a block level up to T10. A total of $12 \mathrm{ml}$ of bupivacaine with $50 \mu \mathrm{g}$ of fentanyl was used to produce the block. Oxygen was administered throughout the procedure at $\mathrm{FiO}_{2}$ of 0.4. During the course of surgery 'artefact filter' on the ECG monitor was disabled and a bipolar electrocautery was used. During the period of surgery, which lasted for $90 \mathrm{~min}$, the patient received $500 \mathrm{ml}$ of lactated Ringer's solution and one unit of packed red blood cell concentrate. During this period he was haemodynamically stable other than two episodes of hypotension which responded to boluses of fluid and $3 \mathrm{mg}$ ephedrine.

At the end of surgery the patient was shifted to post anaesthesia care unit and the AICD was reactivated. He was shifted to the intensive care unit (ICU) and postoperatively epidural infusion was started with bupivacaine $0.125 \%$ and fentanyl $2 \mathrm{ug} / \mathrm{ml}$ at the rate of $3 \mathrm{ml} / \mathrm{h}$. His stay in ICU for $48 \mathrm{hrs}$ was uneventful and he was subsequently discharged from the hospital after 7 days.

\section{Discussion}

In the United States sudden cardiac death (SCD) accounts for an estimated 350,000 lives lost per year and electrophysiological disorders account as a major cause of SCD. ${ }^{3,4}$ AICD is proving to be effective for treatment of malignant ventricular arrhythmias and hence reducing mortality rate from $66 \%$ to $8.5 \% .{ }^{5}$ Current evidence supports use of AICDs for both primary and secondary prevention of sudden death in patients with ischaemic cardiomyopathy with left ventricular dysfunction with ejection fraction $<35 \%-40 \%$. ${ }^{6}$

Perioperative concerns in patients of AICDs for non cardiac surgeries include a thorough preoperative assessment, evaluation and optimization of any associated co-morbid illness. Malfunctions specific to AICDs include inappropriate or ineffective shock delivery, failure and interactions with drugs or devices affecting the efficacy of therapy. ${ }^{7}$ In general, all ICDs should be deactivated during surgery as they can inappropriately discharge due to signals like myopotentials (due to shivering, fasciculations due to suxamethonium, diathermy, orthopaedic instrumentation or false sensing due to sinus tachycardia or rapid atrial fibrillation). ${ }^{8,9}$ Unlike a pacemaker, the bradycardia pacing operation of an ICD is not forced to pace asynchronously with magnet placement, and, therefore, electromechanical interference (EMI) exposure could still cause pacemaker dysfunction. Our patient had the ICD deactivated during the course of surgery.

There is no consensus on application of magnet during surgery. Use of magnet in the perioperative period is fraught with problems. Magnets can be used to protect the pacemaker dependent patient during EMI. But, not all pacemakers switch to asynchronous mode on the application of magnet and the new generation pacemakers are relatively immune to magnet application and may not convert pacemaker to asynchronous mode. Also, constant magnet application over the pacemaker may lead to continuous or transient loss of pacing. A new programme may be automatically set and yet may not be evident until the magnet is removed. Some AICDs, however, can be programmed to ignore magnet placement using manufacturer's guidelines. ${ }^{10}$ Our patient had magnet placed to convert the intracardiac device to asynchronous mode.

Intraoperative monitoring must include mandatory monitoring with special attention to disable artefact filter in electrocardiographic monitoring as it will allow detection of pacemaker discharge. Preoperative placement of external pacing pads in an anterior-posterior configuration is also 
recommended. Our patient had sterile external pacing pads applied prior to surgery.

Special attention should be paid to causes of EMI as even reprogramming or magnet application will not protect a device and internal circuitry from damage. However it can be reduced with use of bipolar diathermy or an ultrasonic scalpel particularly if the surgery is within $15 \mathrm{~cm}$ of the generator. If monopolar electrosurgical diathermy must be used then only with minimal power setting, 'cutting' rather than 'coagulation' current, and short, intermittent and irregular (e.g., less than $1 \mathrm{~s}$ in duration) diathermy bursts are recommended. ${ }^{11}$ A common error could be to place the grounding pad under the calf or buttock as default; rather, it should be placed in such a way that the current flow will not intersect the pacing system. In our patient, diathermy plate was positioned more than $15 \mathrm{~cm}$ away from the AICD site and the surgeon used bipolar surgical cautery.

If ventricular tachycardia or ventricular fibrillation develops, first eliminate all sources of EMI and if a magnet is in place, then it needs to be simply removed (at least $60 \mathrm{~cm}$ away from the device) to restore previously programmed detection and therapy settings. If the device has been programmed off preoperatively, it has to be reprogrammed. If all fails, external shock must be performed, by placing the defibrillator pads or paddles at least $15 \mathrm{~cm}$ from the device in an anterior-lateral or anterior-posterior position. Importantly, clinically appropriate energy output should be used regardless of the presence of ICD. Patients who receive external shock must have their devices interrogated after surgery ${ }^{11,12}$

Patients with cardiomyopathy with low ejection fraction can pose significant perioperative challenges to the anaesthetists. The commonest problems encountered are heart failure, arrhythmias (ventricular tachycardia and atrial fibrillation) and embolic episodes. Our patient received ACE inhibitors, anticoagulants and antiarrhythmic agents. Patients with low cardiac output need to be monitored for changes in blood pressure in the intra operative period. We therefore had a direct arterial line placed prior to surgery. Our patient also received graded epidural with a combination of bupivacaine and fentanyl to prevent precipitous fall in blood pressure.
Although the pacemaker with AICD was inserted two years back, we did not place central venous cannula as an added safety precaution for lead dislodgement. In the perioperative period hypoxaemia, hypercapnia and inadequate analgesia (which increase sympathetic activity and predispose to ventricular tachycardia or fibrillation) should be taken care of. We had placed an epidural catheter preoperatively and provided continuous epidural with low dose of bupivacaine and fentanyl for effective postoperative analgesia.

In the post anaesthesia period, the potential risks to the ICD include its reprogramming, temporary inhibition of its pacing functions or permanent disablement. ${ }^{14}$ It is essential to monitor such patients post operatively, preferably in ICU, till the vital signs are stable and ICD function is appropriate. Use of graded epidural with narcotics remains a tangible anaesthetic technique for safe management of patients with AICDs.

In conclusion, a thorough knowledge of AICD programming, communication with the cardiologist and a clear understanding of the manufacturer's guidelines is warranted for the safe conduct of anaesthesia for these high risk cases. Use of regional techniques, appropriate monitoring, strict vigilance and good team work can go a long way in the successful management of these high risk patients.

\section{References}

1. De Marxhena E, Chakko S, Fernandez P. Usefulness of the automatic implantable cardioverter defibrillator improving survival of patient with severely depressed left ventricular function associated with coronary artery disease. Am J Cardiol 1991; 67: 812-6

2. Allen M. Pacemakers and implantable cardioverter defibrillators. Anaesthesia 2006; 61:883-90

3. Gillum RF. Sudden coronary death in the United States: 1980-1985. Circulation 1989; 79:756-65

4. Harrison D. Do ventricular arrhythmias predict sudden death? In: Conray E, Swan HJC, eds. Clinical strategies in ischemic heart diseases. Baltimore, MD: William and Wilkins;1979: pp 107-15

5. Mirowski M. The automatic implantable Cardioverter defibrillator: an overview. Am J Cardiol 1985;6:461-5 
6. Rozner MA. Implantable Cardiac Pulse Generators: Pacemakers and CardioverterDefibrillators. In: Miller RD. ed. Miller's Anesthesia, $7^{\text {th }}$ ed. Philadelphia, PA: Elsevier, 2010; pp 1387-409

7. Gaba DM, Wyner J, Fish J. Anaesthesia and the automatic implantable Cardioverter/defibrillator. Circulation 1993; 87: 1152-68.

8. Kamalvand K, Gill JS. The implantable cardiac defibrillator. Br J Hosp Med 1996;55:37-41

9. Higgin GL. The automatic implantable defibrillator: management issues relevant to the emergency care provider. Am J Emerg Med 1990;8:342-7

10. Rastogi S, Goel S, Tempe DK, Virmani S. Anaesthetic management of patients with cardiac pacemakers and defibrillators for noncardiac surgery. Ann Card Anaesth 2005; 8: 21-32

11. Stone ME, Apinis A. Current perioperative management of the patient with a cardiac rhythm management device. Semin Cardiothorac Vasc Anesth 2009;13: 31-43

12. Colquhoum M, Jones S, Clarke T, et al. Emergency management of arrhythmias and/or shocks in patients with implantable cardioverter defibrillators (ICDs). A statement on behalf of the Resuscitation Council (UK), Heart Rhythm UK (formerly the British Pacing and Electrophysiology Group, BPEG),the Joint Royal Colleges Ambulance Liaison Committee (JRCALC) and the Ambulance Services Association (ASA). Resuscitation 2006; 71:278-282

13. ACLS Provider Manual. Edited by Cummins RO. Dallas, American Heart Association, 2003

14. Schoenfield MH. Contemporary pacemaker and defibrillator device therapy: challenges confronting the general cardiologist. Circulation 2007; 115: 638-53

\section{THE COLLEGE OF ANAESTHESIOLOGISTS OF SRI LANKA}

\section{FREE PAPERS :}

The rules of the competition are:

1. Open to all medical practitioners in Anaesthesia.

2. Duration of the presentation must be no longer than 15 minutes.

3. The work in whole or in part should not have been presented or published elsewhere.

4. An abstract of not more than 200 words should be submitted before the closing date.

5. The first right of publication of the entries submitted lies with the editor of the College of Aanesthesiologists of Sri Lanka.

6. The decision of the panel of judges in all matters relating to the competition including questions relating to eligibility shall be final.

7. The prize will not be awarded if the Judges decide the presentation is not up to standard.

The closing date for the 2013 competition is $31^{\text {st }}$ October 2012. The abstract should reach the undersigned before the closing date. Any expenditure incurred in the preparation of the slides for papers that were selected for presentation will be reimbursed by the College. 3 copies of the full text should be submitted on or before $31^{\text {st }}$ October 2012 to the anaesthetic office. 